\title{
Attack of the snowclones: A corpus-based analysis of extravagant formulaic patterns
}

\author{
STEFAN HARTMANN \\ University of Düsseldorf \\ TOBIAS UNGERER \\ University of Edinburgh
}

\begin{abstract}
The concept of 'snowclones' has gained interest in recent research on linguistic creativity and in studies on extravagance and expressiveness in language. However, no clear criteria for identifying snowclones have yet been established, and detailed corpus-based investigations of the phenomenon are still lacking. This paper addresses this research gap in a twofold way: On the one hand, we develop an operational definition of snowclones, arguing that three criteria are decisive: (i) the existence of a lexically fixed source construction; (ii) partial productivity; (iii) "extravagant" formal and/or functional characteristics. On the other hand, we offer an empirical investigation of two snowclones that can be considered 'prototypical' on the basis of previous literature, namely [the mother of all $\mathrm{X}]$ and $[\mathrm{X} B E$ the new $\mathrm{Y}]$. We use collostructional analysis and distributional semantics to explore the partial productivity of both patterns' slot fillers. In sum, we argue that the concept of snowclones, if properly defined, can contribute substantially to our understanding of creative language use, especially regarding the question of how social, cultural, and interpersonal factors influence the choice of more or less salient linguistic constructions.
\end{abstract}

Keywords: Snowclones, extravagance, phraseology, Construction Grammar, creativity

\section{INTRODUCTION}

The concept of 'snowclones' has received increased attention in recent constructionist approaches to language (e.g. Traugott \& Trousdale 2013, Traugott 2014, Bergs 2018, 2019). Traugott \& Trousdale (2013: 150) define snowclones as 'schemas that grow from relatively fixed micro-constructions that are usually formulae or clichés'. Well-known examples include [X BE the new Y] or [the mother of all $\mathrm{X}]$. While snowclones have become a relatively popular concept in research on the emergence of linguistic constructions (constructionalisation) and in studies on linguistic creativity, the hedges used in Traugott \& Trousdale's definition (relatively fixed; usually) indicate that the term is used rather vaguely in the current literature. To our knowledge, no clear definition offering reliable criteria for identifying snowclones has been put forward so far, which also raises the question of whether the concept is a useful one in the first place. The main goal of this paper is to refine the concept of snowclones by offering a critical review of previous approaches on the one hand and a detailed analysis of the two patterns that can arguably be considered the most 'prototypical' snowclones, viz. [X $B E$ 
the new $\mathrm{Y}$ ] and [the mother of all $\mathrm{X}$ ], on the other. Analysing these constructions can help us understand whether and how 'snowclones' differ systematically from other constructional patterns.

Snowclones have often been discussed in the context of linguistic extravagance, which is another topic that enjoys growing popularity in the field (see e.g. Petré 2017, De Wit et al. 2020, Ungerer \& Hartmann 2020, Eitelmann \& Haumann 2022). Snowclones are obviously perceived as constructions that 'stand out' in some way. Understanding snowclones thus also entails understanding why they are perceived as 'extravagant'. In the remainder of this paper, we will first provide a brief overview of previous research on snowclones (Section 2). Based on the extant literature, we will then propose a number of criteria for defining the concept (Section 3). In Section 4, we discuss our corpus-based case studies, focusing on the semantic profiles of the patterns in question. Section 4 brings together the theoretical and empirical findings and points to some open questions that should be addressed in future research.

\section{SNOWCLONES: A BRIEF HISTORY}

The term 'snowclone' was coined in the early 2000s in a 'naming contest' initiated by Geoff Pullum on the linguistics blog Language Log (Pullum 2004). Pullum prompted the community to come up with a suitable label for 'a multi-use, customizable, instantly recognizable, time-worn, quoted or misquoted phrase or sentence that can be used in an entirely open array of different jokey variants by lazy journalists and writers' (Pullum 2003b). The term 'snowclone' was suggested by Glen Whitman with reference to Pullum's original example, the journalistic trope that 'if Eskimos have $\mathrm{N}$ words for snow, $\mathrm{X}$ surely have $\mathrm{Y}$ words for $\mathrm{Z}$ '. What makes this pattern a snowclone is the fact that it contains several open slots which can be instantiated by varying lexical fillers, as in (1).

(1) If Eskimos have dozens of words for snow, Germans have as many for bureaucracy (qtd. in Pullum 2003a)

Since then, more than a hundred Language Log posts have been devoted to identifying other snowclones. Moreover, several online collections of snowclones have been created (e.g. O'Connor 2007, Dammerer 2007, and the languagespecific Wikipedia entries for 'snowclone'), which between them comprise several dozens of snowclones from different languages, especially English, German and French. What these early discussions - as well as more recent case studies such as Tizón Couto's (2021) analysis of COVID snowclones have in common is that they consider snowclones on a case-by-case basis, illustrating each pattern with a range of examples collected via informal web searches, but mostly focusing on their suspected cultural and etymological origins. Little attention has been given to common definitional characteristics that would set snowclones apart from other semi-fixed idiomatic patterns, as well as their theoretical treatment within the context of existing constructionist and 
phraseological research. A notable exception is Zwicky's (2007) blog post, in which he proposes a four-step process of how snowclones emerge. According to this account, the creation of snowclones involves: (i) a pre-formula stage during which an idea is expressed in various alternative ways; (ii) a first 'fixing' stage during which the idea is captured by a memorable, lexically fixed phrase; (iii) a third stage in which the pattern is extended by the insertion of open slots or playful allusions to it; and (iv) a second 'fixing' stage in which the variants become (relatively) routinised into a partially fixed schema with open slots.

In more recent years, several researchers have called for a more systematic, data-driven inquiry into the theoretical status and empirical scope of snowclones. Dancygier \& Vandelanotte (2017b) argue that snowclones should be treated as a distinct construction type with specific formal, semantic and multimodal features within the framework of Construction Grammar, rather than leaving them 'filed, unanalysed, under "lazy journalists" clichés'. This, they propose, would allow researchers to 'tap into a quickly growing resource of usage whose popularity calls for a linguistic explanation, relying on various analytical tools (qualitative and quantitative alike)'. In a similar vein, Traugott \& Trousdale (2013; see also Traugott 2014; Traugott \& Trousdale 2014) regard snowclones as a distinct class of idiomatic clausal constructions and discuss their emergence as a paradigm case of lexical, or 'contentful', constructionalisation. In contrast to traditional accounts of lexicalisation as morphophonological reduction of lexical forms, they argue that contentful constructionalisation involves the creation of partial schemas through processes of both expansion and reduction. Snowclones, under this view, illustrate particularly clearly the process of schema expansion as they arise from inserting variable lexical slots into previously fixed patterns.

Other aspects that have sparked recent research interest are the ways in which snowclones illustrate the interplay of linguistic flexibility and rigidity for the purposes of expressing 'old concepts anew' (Hill 2018), and their role as markers of linguistic creativity (Bergs 2018, 2019). Bergs' (2019) characterisation of snowclones as instances of what he calls 'E-creativity' - which is 'what we see when somebody breaks the rules and does something that is not part of the finite set of rules' (Bergs 2019: 175) - also emphasises the status of snowclones as highly salient, or 'extravagant', constructions. As mentioned in the Introduction, the concept of linguistic extravagance has been re-discovered in the recent literature, especially in diachronic Construction Grammar. According to Haspelmath's (1999) original proposal, the term 'extravagance' captures one of Keller's (1994) 'maxims of action': 'Talk in such a way that you are noticed.' On this view, then, snowclones can be considered instances of innovative language use that - at least partly - aims at drawing attention to the speaker.

While the above approaches have contributed to a more theoretically informed investigation of snowclones, they are still limited by (i) the lack of a comprehensive definition of snowclones that provides an overview of their prototypical characteristics, and (ii) the lack of quantitative corpus-based evidence in support of the theoretical claims, especially regarding the productivity and semantic 
flexibility of specific snowclones. We will address both of these issues in turn in the following sections: In Section 3, we will develop a criteria-based definition of snowclones based on previous suggestions in the literature and their extensions. In Section 4, we will present corpus-based case studies on the productivity of two selected snowclones.

\section{DEFINING SNOWCLONES}

While several definitions of 'snowclones' have been proposed in previous work, no attempt has yet been made to merge the suggested criteria into a comprehensive and operationalisable account. Below, we will therefore examine a number of previous proposals, and extract their common characteristics. By combining them and adding some further modifications, we will arrive at a definition of snowclones that is based on three prototypical criteria. To start with, compare the four previous definitions of snowclones in (2):

(2) (a) 'crisp phrasal templates, with one or two open slots to be filled, generalizing a well-documented specific quotation' (Liberman 2006)

(b) 'schemas that grow from relatively fixed micro-constructions that are usually formulae or clichés' (Traugott \& Trousdale 2013: 150)

(c) 'patterned figures of speech that empower swapping out words, phrases, or images for one another without breaking the original pattern' (Hill 2018: 423)

(d) 'a cliché pattern or frame that gives rise to many slightly different variations of this pattern' (Bergs 2019: 176-177)

One common feature pointed out by all these statements is that snowclones derive from a lexically fixed source construction. This characteristic functions as the first of the three characteristics captured by our definition below. As illustrated by the examples in Table 1 , the source instances are typically quotations from real-life historical figures, memorable lines from cultural products (books, songs, films, video games, etc.), or slogans from commercial advertisements. The source constructions need to be culturally salient enough to be instantly recognisable by the speech community in which the snowclone is propagated. This does not mean that the source construction necessarily coincides with the earliest attestation of the pattern. For example, [the mother of all $\mathrm{X}$ ] is usually attributed to a quote by Saddam Hussein from 1991, even though some earlier (but culturally less salient) instances of the pattern can be found in corpora (see Section 4.2). Similarly, source constructions can be effective even if their origins are vague or disputed: for instance, [X BE the new $\mathrm{Y}$ ] can be traced back to popular fashion slogans since the 1970s, irrespective of what exact form these slogans took (e.g. pink is the new black, or a combination of other colours; see Section 4.3). For the emergence of new snowclones, speakers' beliefs about the alleged source of those patterns are of greater relevance than their actual etymological origin. 
Table 1

Examples of snowclones and their (suspected) source constructions.

\begin{tabular}{l|l}
\hline Snowclone & (Suspected) lexically fixed source \\
\hline [the mother of all X] & $\begin{array}{l}\text { the mother of all battles (1991 speech by Saddam } \\
\text { Hussein) }\end{array}$ \\
\hline $\begin{array}{l}\text { [X are from Mars, Y are from } \\
\text { Venus }]\end{array}$ & $\begin{array}{l}\text { Men are from Mars, women are from Venus (1992 } \\
\text { book by John Gray) }\end{array}$ \\
\hline$[\mathrm{I}$ am X, hear me Y] & $\begin{array}{l}\text { I am woman, hear me roar (1971 song by Helen } \\
\text { Reddy) }\end{array}$ \\
\hline [One does not simply VP] & $\begin{array}{l}\text { One does not simply walk into Mordor (2001 film } \\
\text { 'The Lord of the Rings: The Fellowship of the Ring') }\end{array}$ \\
\hline $\begin{array}{l}\text { [I'm not an X, but I play one } \\
\text { on TV] }\end{array}$ & $\begin{array}{l}\text { I'm not a doctor, but I play one on TV (1986 } \\
\text { advertisement for Vick's Formula 44 cough syrup) }\end{array}$ \\
\hline
\end{tabular}

Moving on to the second defining feature conveyed by all of the above definitions, snowclones are characterised by the extension of the source construction to new instances via partial lexical substitution. Snowclones can thus be regarded as semi-schematic constructions composed of both fixed elements (e.g. the mother of all) and open slots (represented by variables such as $\mathrm{X}$ and $\mathrm{Y}$ ). Snowclones can contain one or several variables (see [X BE the new $\mathrm{Y}]$ with two open slots), which can be either filled by single lexemes or entire phrases (e.g. [One does not simply VP] in Table 1). We will argue in Section 4 that analysing these open slots is key to understanding the constructional semantics of a given snowclone.

A third and final feature of snowclones is less explicit in the definitions in (2), but surfaces in expressions like 'crisp phrasal templates' (our highlighting), 'figures of speech' and, arguably, 'clichés'. The intuition behind these terms seems to be that snowclones do not simply employ everyday inconspicuous language to communicate an idea, but that they do so by using particularly effective and stylistically striking language (compare Zwicky 2006, who refers to snowclones as 'especially apt way[s] of expressing [an] idea'). The concept of 'clichés' is related to this notion of rhetorical effectiveness because clichés are usually regarded as fixed idiomatic expressions whose 'stylistic force' (Howarth 1996: 13) leads to their frequent re-use (or overuse). Nevertheless, we would like to avoid the term here due to its definitional vagueness and potential pejorative connotations.

Instead, we suggest that the striking linguistic features of snowclones can be captured by the concept of 'extravagance', which describes speakers' use of 'imaginative and vivid' language 'in order to be noticed' (Haspelmath 1999: 1057) and has recently gained increasing attention in studies on language change, especially within Diachronic Construction Grammar (e.g. De Wit et al. 2020, Kempf \& Hartmann 2022). As argued by Ungerer \& Hartmann (2020), extravagant linguistic expressions also often display other features, such as deviations from linguistic norms and expectations. Both of these characteristics - imaginative language and norm violations - feature frequently in snowclones. 
This is illustrated by the examples in Table 2, which display extravagant formal and/or functional features at different levels of linguistic analysis, ranging from clausal syntax to prosodic structure to individual lexical items.

Table 2

Examples of extravagant formal and functional features in snowclones

\begin{tabular}{lll}
\hline Snowclone & Example & Comment \\
\hline Striking formal features & & \\
\hline [Have X, will travel] & Have tux, will travel & Structural compactness (ellipsis) \\
[All your X are belong to us] & All your history are belong to us & Syntactic violation \\
{$[\mathrm{X}$ 2: Electric Boogaloo] } & Breakin' 2: Electric Boogaloo & Prosody (rhythm and rhyme) \\
{$[\mathrm{I} \vee \mathrm{X}]$} & $I \bullet N Y$ & Multimodality \\
\hline Striking functional features & & Contradiction/paradox \\
\hline [When I say X, what I really mean is Y] & When I say 'today' what I really mean is 'tomorrow' & Hyperbole \\
[the mother of all X] & the mother of all battles & Lexical ambiguity \\
{$[$ Save an X, ride a Y] } & Save a horse, ride a cowboy & Register/style (pragmatic indexing) \\
\hline I, for one, welcome our X overlords] & I, for one, welcome our new insect overlords & \\
\hline
\end{tabular}

In summary, by combining previous suggestions from the literature with some novel extensions, we propose a definition of snowclones that rests on three key criteria:

Snowclones are a class of partially filled constructions characterised by

(i) the existence of an (alleged) lexically fixed source construction that is culturally salient and has sufficiently high token frequency to serve as a template for snowcloning;

(ii) productivity (operationalised via type frequency), i.e. the extension of the pattern to new instances via lexical substitutions in one or more variable slots;

(iii) distinctive ('extravagant') formal and/or functional characteristics which function as markers of linguistic innovation and increase the pattern's memorability.

Two further comments are in order with respect to this definition. Firstly, the criteria should be understood as prototypical characteristics of the category 'snowclones' rather than as necessary and sufficient conditions. This means that while we do expect snowclones to display all three characteristics to a certain extent, the relative weights of the criteria may differ between individual examples. For example, [X $B E$ the new $\mathrm{Y}]$ is attested with an extensive set of slot fillers (see Section 4.3), thus satisfying particularly clearly criterion (ii). As outlined above, however, its lexically fixed source - criterion (i) - is less well established than in the case of other snowclones. While in other cases like [In $\mathrm{N}$ no one can hear you VP], the source can be clearly pinned down (here: In space no one can hear you scream, the famous tagline of the 1979 film 'Alien'), the emergence of [X $B E$ the new Y] can probably be attribued to fashion slogans like pink is the new black (see Section 4.3 below), which most users of the pattern are most likely unaware of. Similarly, [X BE the new Y] can be regarded as semantically extravagant - criterion (iii) - due to the fact that it typically 
juxtaposes two semantically incompatible or at least non-trivially related concepts (e.g. antifacism is the new facism). Nevertheless, the degree of extravagance is arguably lower than in the case of stylistically marked snowclones such as [I, for one, welcome our $\mathrm{X}$ overlords]. This example also points to a potential tension among the three criteria above. As well-established snowclones like [X $B E$ the new $\mathrm{Y}]$ increase in productivity, they may partially emancipate themselves from their source construction and simultaneously lose some of their perceived extravagance. Alternatively, they may have been perceived as less extravagant from the start, which then facilitated their spread.

Secondly and despite these caveats, we argue that the combination of the three criteria can help delineate snowclones from other types of idiomatic constructions that have been previously discussed in the constructionist and phraseological literature. The distinction from proverbs (e.g. Birds of a feather flock together) and other lexically fixed or 'substantive' (Fillmore et al. 1988) idioms (e.g. kick the bucket) is relatively straightforward based on criteria (i) and (ii) above: these idioms do not contain open slots but only appear in a single lexical form, which cannot be traced back to a historically or culturally salient quotation. A trickier distinction is the one between snowclones and other partially filled constructions like [the X-er the Y-er] (Culicover \& Jackendoff 1999) or [What's $\mathrm{X}$ doing Y] (Kay \& Fillmore 1999), which have been discussed under varying labels such as 'formal idioms' (as opposed to 'substantive'; Fillmore et al. 1988), 'constructional idioms' (Booij 2002) and 'phraseme constructions' (Dobrovol'skij 2013). Snowclones could indeed be regarded as a subtype of these constructional idioms (as suggested by Sailer 2013) given that both classes of expressions fulfil criterion (ii), combining fixed lexical elements with variable slots that give rise to the (partial) productivity of the patterns. Nevertheless, the additional criteria (i) and (iii) set snowclones apart from other constructional idioms: neither can constructions like the X-er the Y-er be traced back to a lexically fixed source, nor do they typically display extravagant formal and functional characteristics to the same degree as observed for snowclones.

In the case studies in Section 4, we will illustrate the three characteristics discussed above by analysing two selected snowclones. Our focus will be particularly on the degree of productivity and semantic variability of the respective patterns (criterion (ii) above), but we will also look for evidence of their lexical source (criterion (i)) as well as their extravagant characteristics (criterion (iii)).

\section{CAse Studies}

In this section, we present two case studies on English snowclones. We chose two constructions that are frequently mentioned as typical examples of snowclones (Zimmer 2007, Traugott \& Trousdale 2013: 150): [the mother of all X] and [X BE the new $\mathrm{Y}]$.

The goal of our analysis is to examine to what extent our two selected snowclones display the three characteristics outlined in Section 3. To do so, we 
analyse the range and semantic type of the lexical items that are attested in the open slots of the snowclones. First, we will assess whether the slot fillers that combine most typically with the snowclones provide evidence of a (putative) lexically fixed source for each pattern (criterion (i) from Section 3). Second, we will explore the degree of productivity of the snowclones (criterion (ii)). Here, we will aim to characterise both the semantic range of the patterns, as well as the extent to which they are semantically constrained. We will conduct the analysis both on a global level (e.g. using type/token ratios) and on the level of specific semantic clusters that comprise similar slot fillers. Where the sparse data allow for it, the analysis can also be extended to diachrony. In the case of [X $B E$ the new Y], we will additionally assess the relationship between the two open slots (X and Y). This can not only provide us with further insights about the semantic profile of the snowclone, but it may also reveal whether the snowclone has spawned productive sub-constructions that only contain a single open slot. Finally, we will look, at least tentatively, for signs that the two snowclones encode features of extravagant language (criterion (iii)). While there are no agreedupon ways of 'measuring' extravagance (see Ungerer \& Hartmann 2020), the semantics of the slot fillers may provide at least implicit evidence of whether the snowclones feature inconspicuous semantic profiles, or whether they display unusual or imaginative characteristics.

\subsection{Data and methods}

For both snowclones, we queried three different corpora: The Corpus of Historical American English (COHA, Davies 2010), the Corpus of Contemporary American English (COCA, Davies 2008), and the webcorpus ENCOW16B (Schäfer \& Bildhauer 2012, Schäfer 2015). While the data from the two former were taken into account exhaustively, we worked with samples of 5,000 instances each in the case of ENCOW. False hits were manually excluded (see the individual case studies for the criteria according to which we identified the instances of each construction). Table 3 shows the number of hits for each construction in each corpus along with information about corpus size and the time periods covered by the corpora. As the data from COHA are relatively sparse (which can be seen as an indicator that both patterns are relatively recent innovations), we will mainly focus on the COCA and ENCOW data and only use COHA for a qualitative assessment of early examples.

Table 3

Overview of the corpora and the number of hits.

\begin{tabular}{lllrr}
\hline Corpus & No. of tokens & $\begin{array}{l}\text { Time } \\
\text { period }\end{array}$ & Hits for [the mother of all X] & Hits for [X BE the new Y] \\
\hline COHA & $400 \mathrm{~m}$ & $1810-2009$ & 88 & 6 \\
COCA & $560 \mathrm{~m}$ & $1990-2017$ & 211 & 82 \\
ENCOW & $16.8 \mathrm{bn}$ & Web corpus & 4,127 & 3,848 \\
\hline
\end{tabular}


Before we turn to the case studies, we briefly outline the methodology employed for both studies. For each pattern, we will first look at the 'etymology' of the snowclone, taking into account data from COHA and COCA. A quantitative analysis of the patterns' frequency development is combined with a qualitative look at the early data with regard to the semantics of the slot fillers. For a more detailed quantitative analysis of the synchronic use of the pattern, data from ENCOW are used. For a deeper understanding of the semantic tendencies that the patterns exhibit, we investigate their open slots using simple frequency counts as well as two explorative methods, both of which have come to be widely used in constructionist approaches to language variation: Collostructional analysis on the one hand and distributional semantics on the other.

Collostructional analysis is a family of methods that allows for quantifying the relationship between constructions (see Stefanowitsch 2013 for an overview). In the present study, we use the following methods from the family of collostructional analyses:

- Simple collexeme analysis (Stefanowitsch \& Gries 2003), which identifies association patterns typically between lexical items and syntactic constructions (but see Hartmann 2014 and Smirnova 2021 for morphological application variants). For each lexical item $\mathrm{i}$ in a syntactic construction $\mathrm{C}$ with one open slot, a cross-tabulation test is computed over a $2 \times 2$ table containing a) the frequency of $\mathrm{i}$ in $\mathrm{C}, \mathrm{b}$ ) the frequency in which $\mathrm{C}$ is attested with any other lexical item $\neg \mathrm{i}$, c) the frequency of the lexical item $\mathrm{i}$ in all other constructions $\neg \mathrm{C}$, and d) the frequency of all other lexical items $\neg \mathrm{i}$ in all other constructions $\neg \mathrm{C}$. In doing so, one can identify lexemes that occur with above-chance frequency in a construction. These are called 'attracted collexemes', while lexemes that occur much less often than would be expected at chance level are called 'repelled collexemes'. For example, (Stefanowitsch \& Gries 2003) show with the help of a simple collexeme analysis that for the [X waiting to happen] construction, words with negative prosody like accident or disaster are identified as strongly attracted collexemes.

- Covarying collexeme analysis (Stefanowitsch \& Gries 2005), which investigates association patterns between two open slots in the same construction. Given a construction with two open slots $s_{1}$ and $s_{2}$, for each combination of lexical items $l_{1}$ and $l_{2}$, a cross-tabulation test is computed over a 2 E2 table containing a) the frequency with which $l_{1}$ occurs in the first open slot $s_{1}$ and $l_{2}$ in the second open slot $s_{2}, b$ ) the frequency with which any other lexeme $\neg l_{1}$ occurs in the first open slot $s_{1}$ while the second open slot $s_{2}$ is filled with $\left.l_{2}, c\right)$ the frequency with which $l_{1}$ occurs in $s_{1}$ while $s_{2}$ is filled with any other lexeme $\neg l_{2}$, d) the frequency in which both $s_{1}$ and $s_{2}$ are filled with other lexemes $\neg 1_{1} / \neg l_{2}$. Using covarying collexeme analysis, (Stefanowitsch \& Gries 2005) show that in the so-called into-causative (He talked me into writing this paper with him), fool and think are the most 
strongly attracted covarying-collexeme pair.

All collostructional analyses reported on in this paper were performed using Flach's (2017) package collostructions for R (R Core Team 2020).

Importantly, collostructional methods have a number of limitations that should be kept in mind when interpreting their results (see e.g. Schmid \& Küchenhoff 2013, Gries 2019). Firstly, the association measure is unidirectional: For instance, the fact that the lexical item battle is identified as a strongly attracted collexeme in the [mother of all X] construction indicates that mother of all combines with battle with above-chance frequency, but it does not, vice versa, indicate that battle predominantly occurs in this specific construction. Given its very high frequency (see Table 5 below), we can, quite on the contrary, assume that battle is very promiscuous with regard to the constructions it combines with. Secondly, the most frequently used association measures, e.g. the $p$-value of a chi-squared test, a Fisher Exact Test, or log-likelihood values $\left(\mathrm{G}^{2}\right)$, are sensitive to sample size. Thirdly, association measures do not take dispersions into account. Thus, their results have to be interpreted carefully to make sure that they are not artefacts of e.g. the text type composition of a given corpus. Given the explorative nature of the present study, these caveats are not too problematic here, but they should of course be taken into account when interpreting the results.

Apart from collostructional analysis, we use semantic vector spaces. This method has become more and more popular in recent years under the heading of 'distributional semantics' (see e.g. Levshina \& Heylen 2014, Levshina 2015, Hilpert \& Perek 2015, Perek 2016, Perek \& Hilpert 2017). It allows for quantifying the similarities and dissimilarities between words based on their collocates. For example, a word like summer will co-occur with e.g. spring or height, a word like disaster with e.g. victim or environmental. We will follow Levshina (2015: 323-332) in our application of semantic vector spaces: We first compute Positive Pointwise Mutual Information (PPMI) scores from co-occurrence frequencies, using a window of 5 words on the left and 5 words on the right of each target word. We then compute the cosine similarity between the PPMI vectors. For visually representing the results, we use Multi-Dimensional Scaling (MDS), which transforms configurations of at least $n+1$ items in fewer than $n$ dimensions (see Wheeler 2005: 548), which in our case results in a representation of semantic similarities in a two-dimensional space. This in turn allows for, metaphorically speaking, charting the semantic territory of each construction. For the semantic vector-space analysis of [the mother of all $\mathrm{X}$ ], we draw on COCA; given the fairly low number of hits for [X BE the new Y] in COCA, our semantic vector-space analysis of this construction is based on the ENCOW data. 


\section{2. [The mother of all $X$ ]}

Our first case study is devoted to the snowclone [the mother of all $\mathrm{X}$ ], which is used to signal prototypicality or abundance. According to dictionaries of contemporary English, it denotes 'an extreme example of something' ${ }^{1}$ or 'something regarded as the biggest, most impressive, or most important of (its kind) ${ }^{2}$. The lexical source of the snowclone is usually attributed to Iranian dictator Saddam Hussein, who used the term 'mother of all battles' in a televised speech on the eve of the First Gulf War (Ferguson 2019: 202). Previous metaphorical uses indicate linear descent: for instance, Lass (2015: 46) cites Isidor of Seville's assertion that Hebrew should be considered the 'mother of all languages and literatures'. However, the use of [mother of all X] in a genealogical sense, even if used metaphorically, cannot be considered a snowclone in our view as these instances constitute relatively straightforward cases of personification. This is why cases like (3) were discarded. In addition, false hits that obviously do not correspond to the syntactic target structure were filtered out manually, e.g. The judge acquitted Merlin's mother of all charges (ENCOW).
(a) ' $\mathrm{X}$ is a literal or metaphorical descendant of its antecedent': stem cells (which are the mother of all cells - 'mother cells'!) (Cosentino Fabius: First Word, COCA)
(b) ' $\mathrm{X}$ is brought about by its antecedent': Necessity is the mother of all innovation (04884707b18656d2a12f51ce7d55e29bf488, ENCOW)
(c) ' $\mathrm{X}$ is supported/protected by its antecedent': The banks had failed, but the government had stepped in. It became the mother of all banks (7ba947acf40e70ed0d1c79f2aeff99b0505b, ENCOW)

Starting with the results of the corpus analysis, we will first examine some simple measures that are based on the type and token counts for [the mother of all $\mathrm{X}]$. These measures provide a first impression of the overall productivity of the snowclone. Table 4 summarises the number of tokens, types and hapax legomena attested in COCA and ENCOW. Moreover, Figure 1 plots the temporal development of the token frequency, type frequency, type-token ratio, and the proportion of hapax legomena in COCA between 1990 and 2017. Together, the data illustrate that the snowclone has been quite productive since its beginnings. Both the type-token ratio and the proportion of hapax legomena have been consistently high; they even seem to have increased somewhat over the period of investigation. Given the sparseness of the COCA data, the diachronic trends should be viewed with some caution, but they illustrate that the snowclone has overall maintained high levels of productivity.

[1] https://dictionary.cambridge.org/dictionary/english/mother-of-all-sth (last checked
20/07/2020)

[2] https://www.collinsdictionary.com/dictionary/english/the-mother-of-all_1 (last checked 20/07/2020) 
Table 4

Number of tokens, types and hapax legomena attested for [the mother of all $\mathrm{X}$ ] in COCA and ENCOW

\begin{tabular}{lrrr}
\hline & Tokens & Types & Hapax Legomena \\
\hline COCA & 211 & 160 & 138 \\
ENCOW & 4127 & 1669 & 1092 \\
\hline
\end{tabular}
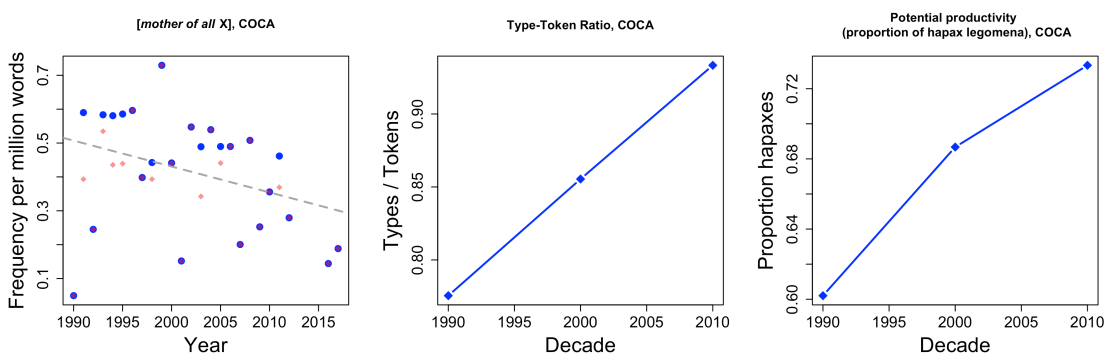

Figure 1

Changes in (normalised) token frequency (big blue dots) and type frequency (small red squares), type-token ratio, and proportion of hapax legomena for [the mother of all $\mathrm{X}$ ] in

COCA.

As a second step, we use simple collexeme analysis to identify the lexemes that are strongly attracted to the open slot of [the mother of all $\mathrm{X}$ ]. This way, we can gain a more detailed understanding of the semantic range covered by the snowclone. Tables 5 and 6 report the top 10 simple collexemes of the construction in COCA and ENCOW, respectively. Two key observations can be made based on these data. Firstly, in both corpora, the most strongly attracted collexeme is battle, thus hinting at the lexically fixed source of the snowclone (see Saddam Hussein's quotation above). While the pattern has been extended to various other slot fillers, none of these have reached the same frequency as the source construction. This suggests that the snowclone has not fully emancipated from its lexical source, which continues to be culturally salient for speakers.

Secondly, the top 10 collexemes indicate certain semantic preferences among the slot fillers. In particular, [the mother of all $\mathrm{X}$ ] seems to combine primarily with abstract concepts that have negative semantic prosody (e.g. storm, crisis, headache, hangover, bailout). Moreover, the slot fillers include a number of everyday concepts (e.g. [traffic] jam, headache) and also colloquial terms (e.g. cockup). The fact that several of the top collexemes describe dangerous or otherwise unpleasant events indicates that speakers use the snowclone in situations in which they are emotionally strongly involved (which, incidentally, is also one of the defining criteria of extravagance that have been offered in the literature, see Petré 2017: 125; see Ungerer \& Hartmann 2020 for a critical discussion of this criterion, 
and see below for a discussion of the extravagance of the mother of all snowclone).

Table 5

Results of the simple collexeme analysis for [the mother of all $\mathrm{X}]$ in COCA

\begin{tabular}{llll}
\hline Collexeme & Frequency in cxn & Total frequency & Collostruction strength $\left(\mathbf{G}^{\mathbf{2}}\right)$ \\
\hline battle & 21 & 45078 & 259.16198 \\
storm & 5 & 31113 & 50.6806 \\
jam & 3 & 9363 & 34.51345 \\
bomb & 3 & 26793 & 28.21755 \\
op & 2 & 2645 & 26.43214 \\
war & 4 & 192993 & 24.26394 \\
network & 3 & 56670 & 23.74499 \\
stickup & 1 & 8 & 23.5558 \\
headache & 2 & 6169 & 23.04645 \\
movie & 3 & 82435 & 21.51553 \\
\hline
\end{tabular}

Table 6

Results of the simple collexeme analysis for [the mother of all X] in ENCOW

\begin{tabular}{llll}
\hline Collexeme & Frequency in cxn & Total frequency & Collostruction strength $\left(\mathbf{G}^{\mathbf{2}}\right)$ \\
\hline battle & 148 & 451149 & 1092.89932 \\
hangover & 62 & 14701 & 772.37606 \\
bubble & 50 & 93674 & 416.34848 \\
crisis & 60 & 493823 & 324.46063 \\
bailout & 28 & 33285 & 258.36918 \\
headache & 29 & 52491 & 243.32028 \\
adventure & 34 & 181243 & 212.58626 \\
cock-up & 15 & 1902 & 205.51346 \\
bomb & 27 & 145083 & 168.34877 \\
storm & 28 & 184353 & 163.4198 \\
\hline
\end{tabular}

The results of a semantic vector-space analysis (as described in Section 3 above) based on COCA are shown in Figure 2. As outlined above, the method of multidimensional scaling allows for representing distances in two-dimensional space (Levshina 2015: 336). Hence, items that are displayed close to one another are identified as similar, based on their collocates, while items that are farther away from each other are seen as rather dissimilar. The font size of the lexemes represents the frequency with which they are attested in the [mother of all $\mathrm{X}$ ] construction in COCA. Exploring the distribution of lexemes on the plot, we can roughly distinguish two major groups and one minor group: battle stands out as the most frequent item in a group of lexemes on the bottom right-hand corner of 
the plot, many of which relate to the military domain (bomb, storm, war, in some contexts also parade). In addition, abstract terms referring to organisations or institutions (union), person collectives (band), and events (festival) can be found in close proximity as well. The second major group clusters in the lower lefthand area of the plot, with headache as its most frequent member. It contains words with a rather negative semantic prosody, many of which belong to the domain of health conditions or risky health behavior (hangover, overdose), but we also find abstract terms like screw-up. The third group with only a few members can be found in the top half of the plot, slightly left from the centre: jam, pork, pizza, sauce, egg, and recipe form a highly coherent group of food-related items. (Note, however, that jam only occurs in the data in the compound traffic jam - for compounds, we only took the heads into account, hence this is not reflected in the vector-space analysis.)

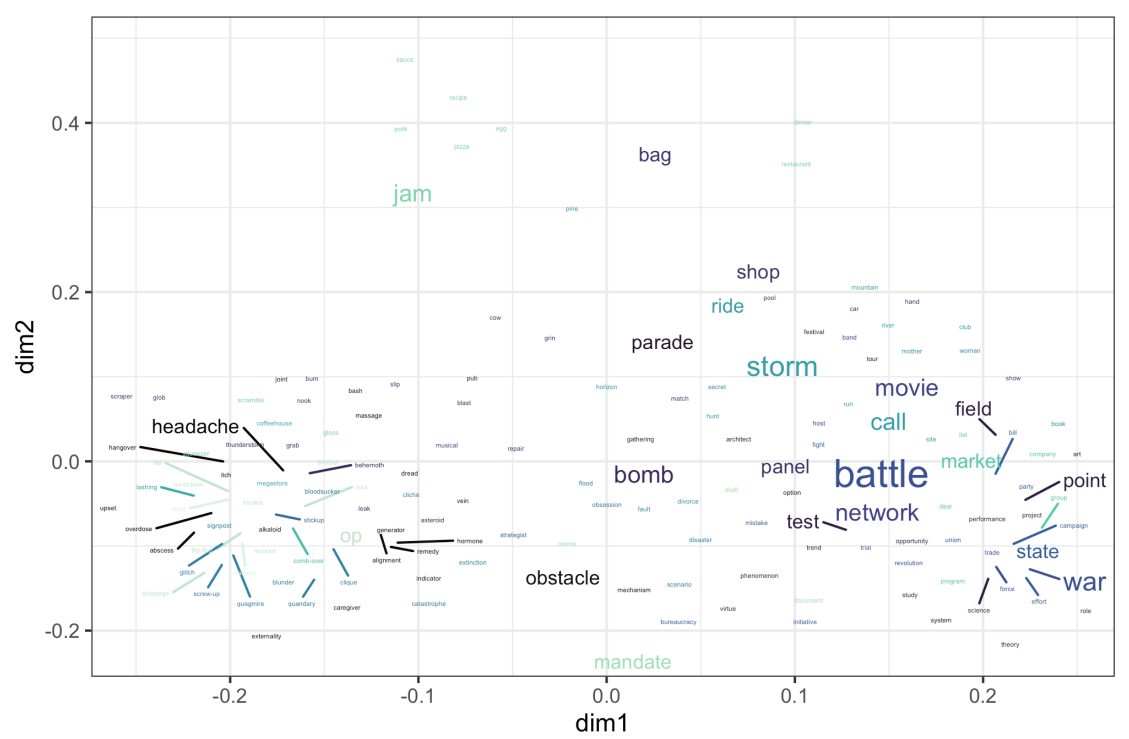

Figure 2

Semantic vector-space analysis of the lemmas occurring in [mother of all X] in COCA.

Together, these observations suggest that while the construction combines with a range of different slot fillers, it is still limited to a certain semantic profile. This partial productivity aligns naturally with the characteristics of snowclones as described in Section 3. While a certain degree of semantic flexibility is needed for the pattern to function as a snowclone, it is still tied somewhat to the semantics (and pragmatics) of the original lexical source. The most frequent items, and also the most central ones according to the collexeme analysis, come from the military 
domain or have a negative semantic prosody. But cases like the mother of all restaurants show that the pattern can also be used in a positive sense, and that the (extravagant) constructional semantics of [the mother of all $\mathrm{X}$ ] can be exploited to convey enthusiasm.

\section{3. [X BE the new $Y$ ]}

Our second case study is concerned with the snowclone [X $B E$ the new $\mathrm{Y}$ ], which is 'used to state $\mathrm{X}$ is now fashionable (or common, etc), where $\mathrm{Y}$ was before ${ }^{3}$. For example, smart is the new young (CBS_SunMorn, COCA) expresses that intelligence has come to play a greater role in a context where young age previously used to be a more decisive factor. The origins of the snowclone are usually attributed to fashion slogans from the 1970s and 1980s (e.g. pink is the new black), even though it is not clear when exactly and with what colour terms the snowclone was first attested (see Zimmer 2006). As mentioned in Section 3, the lexical source of the construction is therefore less clearly established than for other snowclones.

In order to identify instances of the pattern, we searched the corpora exhaustively for the string is/are the new preceded and followed by a token tagged as either an adjective or a noun. ${ }^{4}$ Attestations in which the pattern is used to refer to an identity or a class inclusion relation (Glucksberg \& Keysar 1993: 412), as illustrated in (4), were manually discarded; this also includes metaphorical instances such as (4-c). Doubtful cases were marked as such but not taken into account in the final analysis. For example, nearly all instances of [X is the new religion] can be seen as doubtful cases as it is unclear whether they are used in the sense of ' $\mathrm{X}$ is (like) a religion now', thus constituting a (metaphorical) class inclusion relation, or rather in the sense of ' $\mathrm{X}$ has taken the place of religion' (the snowclone meaning).

(4) (a) Penny Adkins is the new manager of the Imaging unit of Central Services. (Denver Post, People on the Move, 1997, COCA)

(b) Daniel Craig is the new James Bond (0fb25d98cec169aa250051d81 $44 b b 34 c 11 a b$, ENCOW)

(c) after the French macarons, cupcakes are the new scene stealers this year (251fe7457256dc055976e77fb3fcddefb593, ENCOW)

[3] https://www.wiktionary.org/wiki/Appendix:Snowclones/X_is_the_new_Y

[4] It has been claimed that the adjectival pattern $\left[\mathrm{ADJ}_{1} B E\right.$ the new $\left.\mathrm{ADJ}_{2}\right]$ is the construction that gave rise to $[\mathrm{X} B E$ the new $\mathrm{Y}]$ and that the latter was only later extended to nouns (see e.g. Traugott \& Trousdale 2014: 272; also see the blog posts by Zimmer 2006 and Zwicky 2006). However, the $\left[\mathrm{N}_{1} B E\right.$ the new $\left.\mathrm{N}_{2}\right]$ pattern is attested in COHA already in the late $19^{\text {th }} /$ early $20^{\text {th }}$ century, as the following examples show: 'To-day', he sings, 'Milan is the new Athens!' (Julia Cartwright Ady, Beatrice d'Este, Duchess of Milan, 1475-1497, 1899, COHA); The education department of the Government of India is the new Frankenstein, and the higher education is its monster.(The Atlantic Monthly, October 1913, COHA) 
Before delving into the details of the corpus analysis, a few qualitative observations can be made that already illustrate some of the productivity and extravagance of [X $B E$ the new Y]. At its core, the snowclone describes a comparison between the two concepts in the X and the Y slot. Given that, as Cummins (2019: 144) points out, 'pretty much everything is like pretty much anything else in at least some identifiable respect', the snowclone thus invites speakers to draw original and innovative comparisons. In this sense, the snowclone bears similarity to other constructions like [X $B E$ a Y] or [X BE the PROPER NAME of NP], as in She is the Einstein of Cognitive Linguistics. These constructions have been described as metaphorical (see e.g. Glucksberg \& Keysar 1993, Grady 1999: 98) and can also be seen as conceptual blends in the sense of Fauconnier \& Turner (2002). In these cases, 'the metaphor provides a high degree of implicative elaboration' (Glucksberg \& Keysar 1993: 421). Glucksberg \& Keysar's example Xiao-Dong is a Bela Lugosi, for instance, builds on the hearer's knowledge that Bela Lugosi was an actor best known for his portrayal of Dracula. In using him as a reference point, he is basically 'typified', and depending on the context (and their cultural knowledge), the hearer will identify the salient features that the speaker refers to when comparing Xiao-Dong to Bela Lugosi.

Similarly, instances of [X BE the new Y] such as Spielberg is the new Capra (Love Walked In, COCA) require a 'high degree of implicative elaboration' in that the hearer has to establish the tertium comparationis that connects the two directors (which can of course be more than one single feature). Other cases, such as Android is the new Windows, and Apple is about to see the 1990s all over again (b6137beed83ff4cc69f6bf4a58fcfdd9a9b4, ENCOW), tap into an even more complex configuration of shared encyclopedic knowledge. In this example, hearers need to relate the competition between Apple and Microsoft and the success of Microsoft Windows in the 1990s to the present-day competition on the smartphone market. Quite frequently, the comparisons are so innovative that speakers decide to state the tertium comparationis explicitly, as shown in (5).

(a) America is the new Rome. We like physical sports. (75daf596dc219 6a8348667153ba4e8e74f27, ENCOW)

(b) If depression was the disease of the moment in the 1990s, anxiety is the new depression. (5a2d377a41ce4ff55fdf3988091c3e411163, ENCOW)

(c) Google glasses are the new segway - useful if your company pays you to use it but no consumer who is not a creeper is going to buy one. (7f51314f251f58348c69ac6d5ffcbff182cb. ENCOW)

Together, these examples suggest that $[\mathrm{X} B E$ the new $\mathrm{Y}]$ is a productive pattern that tends to attract extravagant combinations of slot fillers. In the following, we will examine to what extent these initial impressions are confirmed and extended by the quantitative corpus analysis.

As in our first case study, we start by examining the token and type frequencies 
of the snowclone. Table 7 summarises the number of tokens, types and hapax legomena in both corpora. The latter two are calculated for pairs of $\mathrm{X}$ and $\mathrm{Y}$ elements as well as separately for the $\mathrm{X}$ slot and the $\mathrm{Y}$ slot. The high type frequency relative to the number of tokens (which corresponds to type-token ratios of 0.87 and 0.73 for the $\mathrm{X}-\mathrm{Y}$ pairs) suggests that $[\mathrm{X} B E$ the new $\mathrm{Y}$ ] is a highly productive pattern. This is also supported by the number of hapax legomena among x-y pairs, which make up between 64 and $78 \%$ of all attested tokens. Interestingly, a comparison between the type frequencies of the two open slots indicates that the $\mathrm{X}$ slot is overall more productive than the $\mathrm{Y}$ slot (type-token ratios of $0.52-0.76$ versus $0.43-0.63$ ). One reason for this may be that one specific $\mathrm{Y}$ filler (black) is particularly frequent and combines with a range of different $\mathrm{X}$ elements (see below).

Table 7

Number of tokens, types and hapax legomena attested for [X BE the new $\mathrm{Y}]$ in COCA and ENCOW

\begin{tabular}{lrr|rrrr}
\hline & \multicolumn{3}{c|}{ COCA } & \multicolumn{3}{c}{ ENCOW } \\
\hline Tokens & \multicolumn{3}{c}{82} & & \multicolumn{3}{c}{3848} & $\mathbf{y}$ \\
\hline Types & $\mathbf{x}-\mathbf{y}$ pairs & $\mathbf{x}$ & $\mathbf{y}$ & $\mathbf{x}$-y pairs & $\mathbf{x}$ & 1651 \\
Hapax & 71 & 67 & 52 & 2805 & 2000 & 1241 \\
legomena & 64 & 60 & 47 & 2479 & 1505 & 124 \\
\hline
\end{tabular}

Following this quantitative assessment of the snowclone's productivity on a global level, we next conduct simple collexeme analyses to gauge the typical semantics of the slot fillers. In this and the following analyses, we only use data from ENCOW because the number of instances in COCA is too small to draw reliable conclusions about the constructional semantics. Tables 8 and 9 provide the top 10 most strongly associated collexemes for the $\mathrm{X}$ and $\mathrm{Y}$ slot, respectively. A first observation is that colour terms are clearly among the most typical fillers in both slots. This provides clear evidence of the lexical source of the snowclone, which seems to have originated from aesthetic comparisons in the domain of fashion. Interestingly, the $\mathrm{X}$ slot displays more variability in the specific colour that is used (e.g., green, pink, black, grey, white), while the $\mathrm{Y}$ slot is by far most often filled by black (and much less frequently by pink). Black occurs a total of 622 times in the Y slot, thus explaining the above observation that the type frequency among the $\mathrm{Y}$ elements is lower than among the $\mathrm{X}$ elements. This could indicate that $[\mathrm{X} B E$ the new $\mathrm{Y}]$ originated from a set of related source constructions, which combined black with a range of different colours.

Apart from colour terms, the simple collexeme analyses suggest that the construction attracts concepts from the internet and (social) media domain (blog, facebook, transparency), as well as group membership terms (nazi, KKK, jews). These semantic fields point to specific functional contexts in which the snowclone is used. On the one hand, [X BE the new $\mathrm{Y}]$ frequently occurs in internet forums 
and online media; it is therefore not surprising that speakers use the snowclone to draw innovative comparisons between technological developments. Beyond that, the use of group membership terms suggests that the snowclone is also used to characterise (groups of) people in potentially stereotypical and/or pejorative ways.

Table 8

Results of the simple collexeme analysis for the X slot of [X BE the new $\mathrm{Y}]$ in ENCOW

\begin{tabular}{llll}
\hline Collexeme & Frequency in cxn & Total frequency & Collostruction strength $\left(\mathbf{G}^{\mathbf{2}}\right)$ \\
\hline green & 91 & 1227752 & 343.75 \\
pink & 40 & 197699 & 227.92 \\
black & 67 & 1743023 & 172.01 \\
small & 77 & 3367848 & 129.71 \\
grey & 27 & 214880 & 128.75 \\
white & 55 & 1884617 & 114.72 \\
gray & 21 & 166299 & 100.3 \\
blog & 38 & 971381 & 98.49 \\
transparency & 19 & 123758 & 97.93 \\
facebook & 27 & 435778 & 92.51 \\
\hline
\end{tabular}

Table 9

Results of the simple collexeme analysis for the Y slot of [X BE the new $\mathrm{Y}$ ] in ENCOW

\begin{tabular}{llll}
\hline Collexeme & Frequency in cxn & Total frequency & Collostruction strength $\left(\mathbf{G}^{\mathbf{2}}\right)$ \\
\hline black & 622 & 1743023 & 4283.22 \\
sexy & 32 & 82298 & 220.02 \\
oil & 57 & 1095176 & 171.82 \\
nazi & 26 & 116573 & 150.29 \\
big & 75 & 2837001 & 137.6 \\
pink & 27 & 197699 & 130.3 \\
cupcake & 14 & 13211 & 124.04 \\
kkk & 12 & 5835 & 122.16 \\
objectivity & 14 & 27026 & 104.14 \\
jews & 29 & 423586 & 101.88 \\
\hline
\end{tabular}

So far, we have relied on our intuitive judgments to assess the semantics of the top 10 most typical slot fillers. In order to see whether our impressions hold across a larger range of slot fillers, and to obtain a more objective measure of semantic similarity, we next analyse the $\mathrm{X}$ and $\mathrm{Y}$ elements using semantic vector spaces. As mentioned in Section 4.1, we follow Levshina (2015) in our operationalisation of distributional semantics, using a window of 5 words on both sides of the keyword. The collocates needed to perform the semantic vectorspace analysis were extracted from the downloadable portions of ENCOW (see 
the supplementary material for details). The results are visualised in Figure 3, which again groups together semantically similar slot fillers close to each other in a two-dimensional diagram. The colours indicate the relative frequency of each word in the $\mathrm{X}$ or the $\mathrm{Y}$ slot: Items shown in focal red are attested in the $\mathrm{X}$ slot exclusively, items in focal blue are only attested in the Y slot, while items shown in different shades of purple occur in both slots.

Overall, three big clusters are apparent in the diagram, which correspond quite closely with the three semantic fields we identified in the simple collexeme analyses. The cluster on the left mostly contains colour terms (e.g., black, green, red, pink); the cluster on the top right consists of internet-, media- and careerrelated concepts (e.g., data, transparency, marketing, twitter); and the cluster on the bottom right encompasses social groups, institutions and ideologies (e.g., gay, republican, church, racism).

Combining the results of the simple collexeme and the semantic vector space analyses, we can observe that $[\mathrm{X} B E$ the new $\mathrm{Y}]$ displays specific semantic preferences. Similar to [the mother of all X], the snowclone thus covers a set of relatively constrained functional niches. Within its limited semantic profile, the construction is highly productive, but it does not seem to display the same degree of semantic flexibility that would be expected from a canonical comparison construction (e.g., [X is like $\mathrm{Y}]$ ). It may be partially due to its constrained applicability, and its association with certain usage contexts (e.g., internet forums), that the snowclone maintains its innovative and extravagant character.

Having outlined the semantic profile of the individual slot fillers, we now turn to how the $\mathrm{X}$ and $\mathrm{Y}$ elements relate to one another. As mentioned at the beginning of this section, snowclones like [X $B E$ the new $\mathrm{Y}$ ] provide an interesting test case to study how the semantics of the two open slots interact with each other. This is particularly relevant since it may allow us to characterise, on a quantitative level, what makes the comparisons between the $\mathrm{X}$ and $\mathrm{Y}$ concepts so innovative, and thus gives the snowclone its unique character.

As before, we approach this question with several methodological tools. First, we manually categorised all slot fillers in the ENCOW data into coarse semantic categories. These categories included abstract concepts (e.g., communism, crazy), concrete objects (e.g., banana, SUV), colours (e.g., blue, black), social groups (e.g., Democrats, Rolling Stones), persons (Al Capone, Obama), locations (Las Vegas, Mexico), and organisations (airline, Google). We then counted how often each semantic category in the X slot co-occurs with each type of Y element. The results are visualised with the help of a heat map in Figure $4 .^{5}$

A first regularity shown by the diagram is that all types of slot fillers occur most frequently with a concept in the other slot that belongs to the same semantic

[5] The colours in the heat map indicate the logarithmised frequency in order to make the visual difference clearer, while the labels show the original frequency values. 


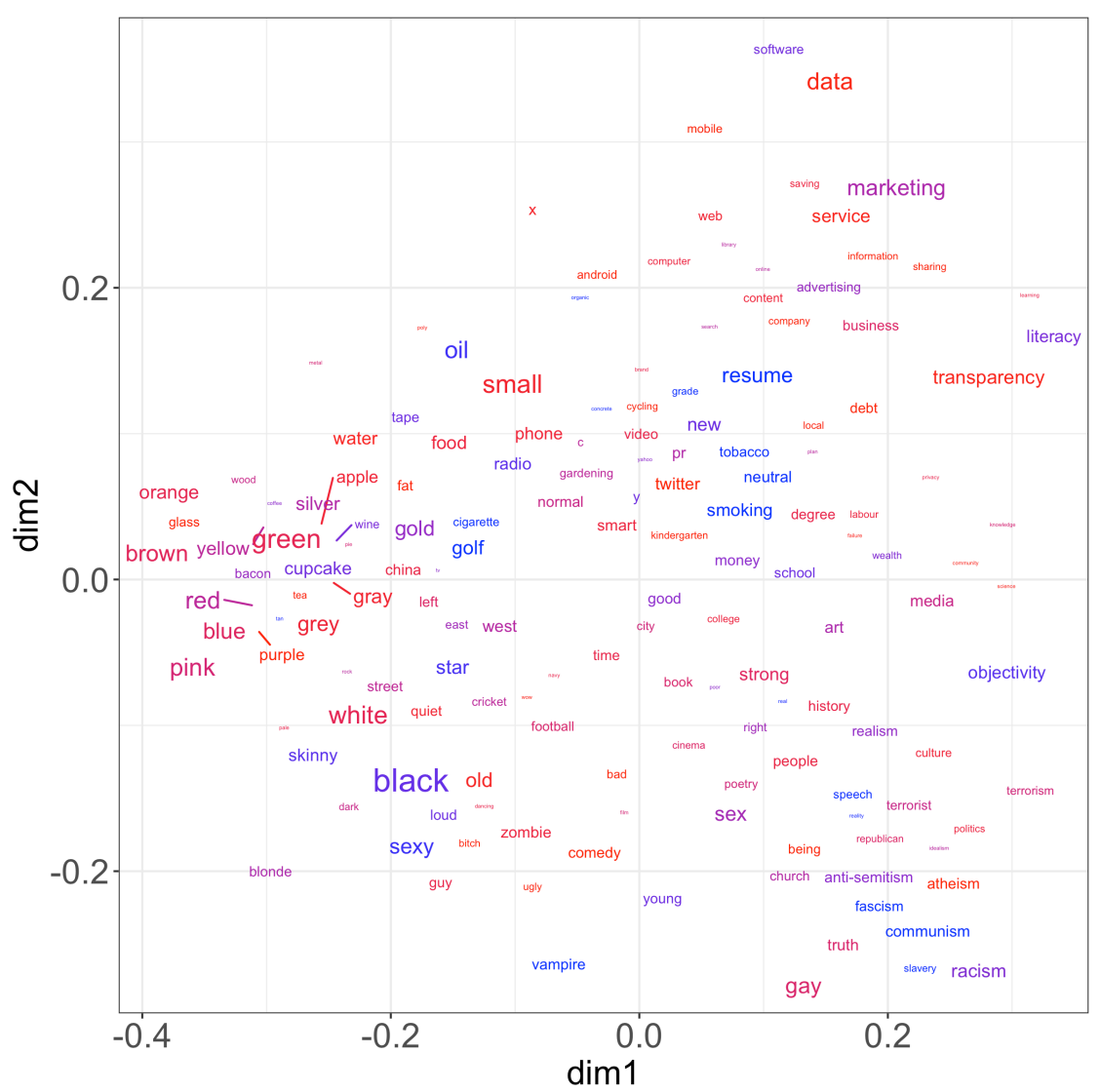

Figure 3

Semantic vector space analysis of the slot fillers in $[\mathrm{X} B E$ the new $\mathrm{Y}]$ based on ENCOW

category. This is not particularly surprising: abstract concepts are typically compared to abstract concepts, locations are compared to locations, and so on. Besides that, however, one other feature deserves attention: abstract $\mathrm{X}$ elements are relatively often paired with concrete concepts or colours in the Y slot (see the first column of Figure 4). These combinations occur much more frequently than the opposite pairings of concrete or colour Xs with abstract Ys. This might point to a specific communicative function of [X $B E$ the new $\mathrm{Y}]$ : the snowclone may sometimes be used to make abstract concepts more tangible and intelligible by relating them to a concrete point of comparison. Examples such as Twitter is the new cigarette (ca2bc545af6cbe8255bcd36968dc7d675c28, ENCOW) or The social network is the new production line (56de85af6ae995d4ecd2f44ea17f77df0ba1, ENCOW) illustrate the impact of social media usage by comparing it to everyday 
concepts from the domains of recreational consumption and manufacturing.

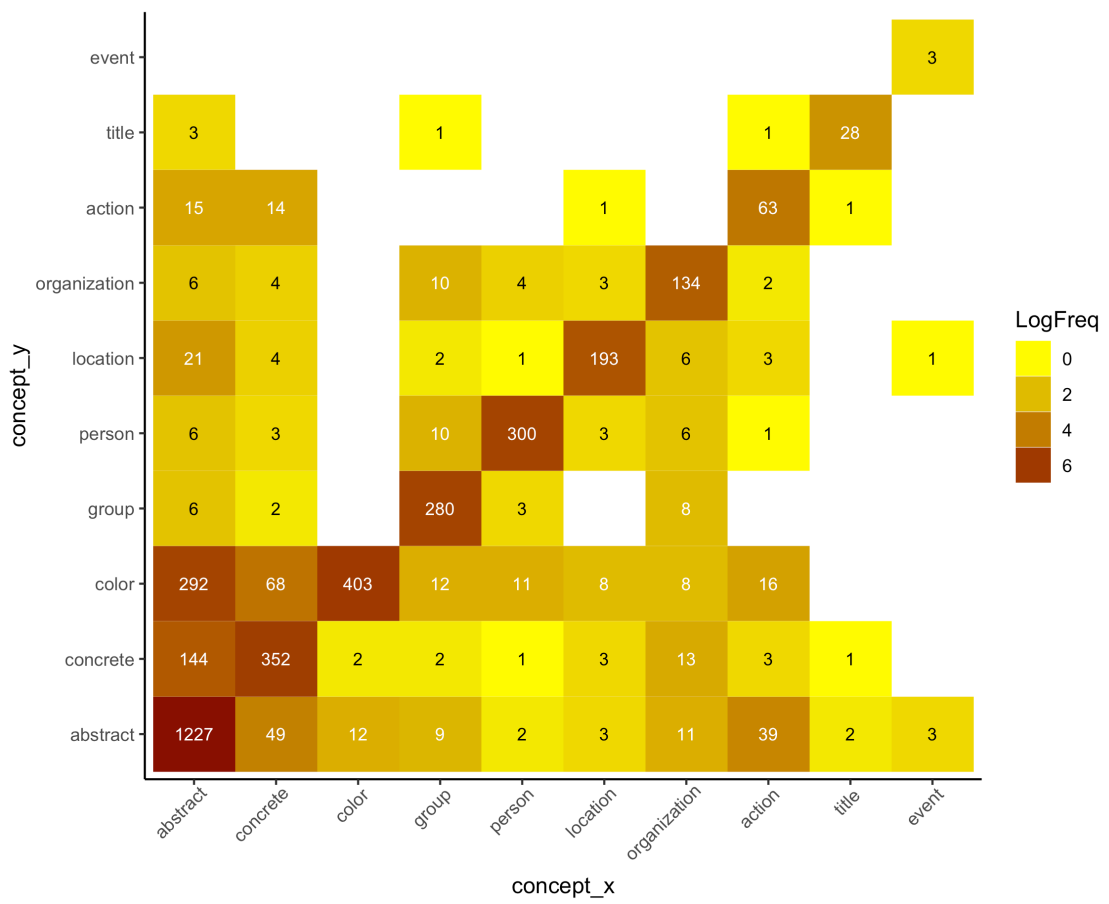

Figure 4

Heat map of semantic categories co-occurring in the X and Y slot of [X BE the new Y] based on ENCOW (the darker the colour, the more frequent the combination).

While the heat map suggests that speakers tend to combine $\mathrm{X}$ and $\mathrm{Y}$ elements from the same semantic category, the question remains whether the comparisons they draw within each category are innovative (and extravagant) or rather conventional. To investigate this question, we first use another type of collostructional analysis - covarying-collexeme analysis - to identify pairs of X and $\mathrm{Y}$ elements that most typically occur together in the snowclone. Table 10 summarises the $\mathrm{X}-\mathrm{Y}$ pairs whose members are most strongly associated with each other. For example, the first row of the table indicates that small and big are almost always combined when they occur in the snowclone 75 times out of 75 and 77 individual occurrences). This example, along with a few other instances (old is the new new, strong is the new skinny, and quiet is the new loud), illustrates that some of the most typical X-Y pairs in the construction consist of (near)antonyms. This provides at least tentative evidence that the snowclone is used to draw 'extravagant' comparisons between seemingly incompatible concepts. For illustration, consider paraphrasing the examples with a canonical comparison 
construction: for example, small is like big or strong is like skinny. Clearly, these sentences sound odd compared with the snowclone examples, suggesting that speakers are more willing to accept unusual combinations when they are presented in the snowclone format.

Table 10

Results of a covarying-collexeme analysis of the [X BE the new $\mathrm{Y}]$ ENCOW data.

\begin{tabular}{llllll}
\hline Slot X & Slot $\mathbf{Y}$ & Freq in Slot $\mathbf{X}$ & Freq in Slot $\mathbf{Y}$ & Combined Frequency & $\mathbf{G}^{\mathbf{2}}$ \\
\hline small & big & 77 & 75 & 75 & 720.65 \\
data & oil & 51 & 57 & 37 & 283.66 \\
blog & resume & 23 & 28 & 20 & 198.98 \\
transparency & objectivity & 19 & 14 & 14 & 163.3 \\
muslims & jews & 18 & 29 & 15 & 139.99 \\
green & red & 91 & 33 & 23 & 138.36 \\
old & new & 23 & 16 & 13 & 126.99 \\
strong & skinny & 11 & 13 & 10 & 118.28 \\
quiet & loud & 8 & 8 & 8 & 114.8 \\
nra & kkk & 9 & 12 & 9 & 113.53 \\
thursday & friday & 13 & 20 & 11 & 112.15 \\
big & small & 8 & 10 & 8 & 104.79 \\
service & marketing & 15 & 26 & 11 & 98.04 \\
google & microsoft & 27 & 21 & 12 & 96.74 \\
bad & good & 7 & 8 & 7 & 96.29 \\
disk & tape & 7 & 8 & 7 & 96.29 \\
black & white & 67 & 20 & 14 & 92.25 \\
x & y & 10 & 7 & 7 & 90.1 \\
atoms & bits & 6 & 6 & 6 & 89.55 \\
kindergarten & grade & 6 & 6 & 6 & 89.55 \\
\hline & & & & &
\end{tabular}

Finally, we examine whether the semantic relation between the $\mathrm{X}$ and $\mathrm{Y}$ slot can be assessed in a more automatised fashion across the whole dataset. For this purpose, we apply again semantic vector spaces, but this time extending the method in a novel (and somewhat tentative) way. Instead of looking at the semantic similarity between different words that fill the same slot, we calculate the cosine similarity between the $\mathrm{X}$ and $\mathrm{Y}$ element in each attested pair. This gives us a measure of the semantic distance between the concepts that are compared in each instance of the snowclone. To illustrate the method, Figure 5 displays the distance scores for a sample of 20 instances that span almost the entire range between minimum and maximum distance (i.e., between 0 and 1). The results align quite well with intuitive judgments: pairings of identical elements are maximally similar (e.g., white-white); subtypes of the same category are quite closely related (e.g., democrat-republican); antonyms display a mid-level semantic distance (e.g., small-large); terms from similar domains without a clear taxonomic or causal relation are more distant (e.g., publishing-literacy); and 
abstract terms that display no featural similarity are highly distant (e.g., fundsblack).

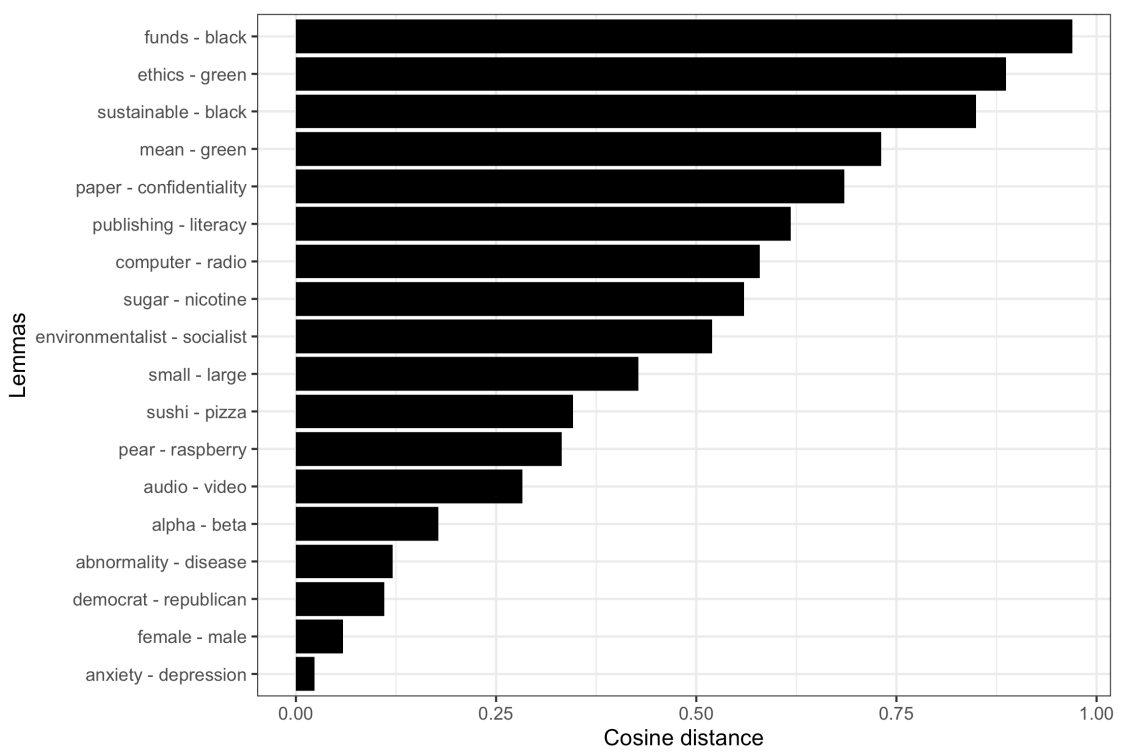

Figure 5

Distribution of semantic distances between $\mathrm{X}$ and $\mathrm{Y}$ elements in [X $B E$ the new $\mathrm{Y}]$ based on ENCOW

Given that the distance measure yields sensible results, we now assess what the typical semantic distance is across all instances of the snowclone. Figure 6 provides a histogram that shows how often the different distance values occur in the data. The results suggest that the semantic distance between $\mathrm{X}$ and $\mathrm{Y}$ most commonly falls into the range between 0.6 and 0.8 (median 0.65). It is, of course, difficult to interpret these values in terms of the 'extravagance' of the $\mathrm{X}-\mathrm{Y}$ pairings, given that no information is available about what the distribution of distance scores would look like for other constructions. Nevertheless, the instances in Figure 5 give an idea of the types of $\mathrm{X}-\mathrm{Y}$ pairs that represent the 0.6-0.8 range (publishing-literacy, paper-confidentiality, mean-green). Other examples from ENCOW are truth-speech, innovation-selfishness, fake-natural and history-cooking. All of these instances seem to encode non-trivial, creative comparisons that may require additional contextual knowledge on the addressee's part. For example, publishing is the new literacy is used to compare the growing opportunities to publish one's own work, especially online, with the way in which literacy, once reserved for a small privileged group, spread to the public. The fact that such innovative comparisons occur frequently in $[\mathrm{X} B E$ the new $\mathrm{Y}]$ hints at its 


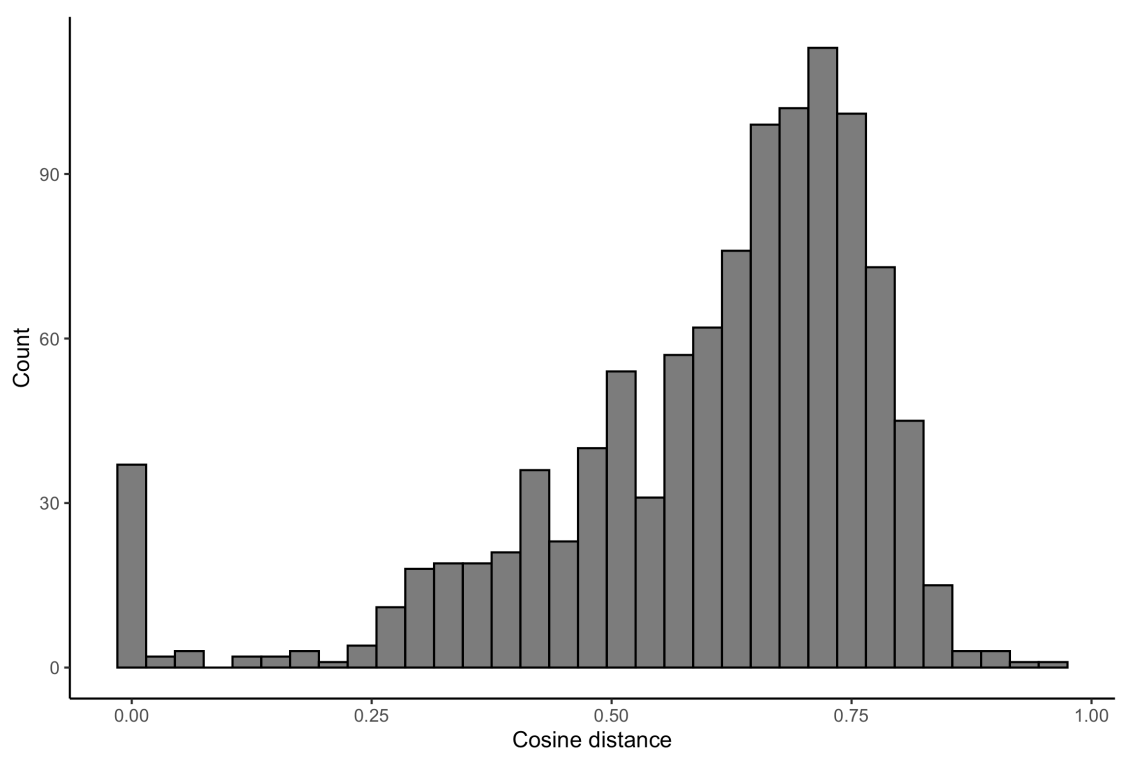

Figure 6

Semantic distance between a sample of $\mathrm{X}$ and $\mathrm{Y}$ elements in [X BE the new $\mathrm{Y}]$ based on ENCOW

extravagant character. Rather than comparing just any kind of consecutive trends or fashions, speakers seem to use the snowclone primarily to highlight interesting commonalities between seemingly disparate concepts.

Summing up, then, our analysis shows that - as in the case of [mother of all $\mathrm{X}$ ] - the productivity of [X $B E$ the new $\mathrm{Y}$ ] is constrained to certain semantic domains, which in themselves are quite heterogeneous, however. As for the combinations of $\mathrm{X}$ and $\mathrm{Y}$ slots, users of the snowclone tend to combine polar opposites (small is the new big) or cohyponyms (Thursday is the new Friday) from the same semantic domain or items from rather different domains, leading to innovative and creative comparisons.

\section{DISCUSSION AND CONCLUSION}

In this paper, we have suggested a definition for snowclones, extravagant formulaic patterns that typically have a lexically fixed source construction and that exhibit distinctive formal and/or functional characteristics. In addition, we have presented an in-depth analysis of two patterns that are frequently discussed as prototypical examples of snowclones, namely [the mother of all $\mathrm{X}]$ and $[\mathrm{X} B E$ the new Y]. We have shown that both patterns recruit their slot fillers from a comparatively limited array of semantic domains: [the mother of all $\mathrm{X}$ ] usually combines with words with negative semantic prosody, often from the military 
domain; however, it can also be used in a positive sense, e.g. when referring to restaurants, food, movies, etc. As such, it can be said that the pattern is used to signal a high degree of emotional involvement. [X $B E$ the new $\mathrm{Y}]$, on the other hand, is somewhat more heterogeneous in that both nouns and adjectives can occur in both of its open slots. Still, its productivity is limited to a certain set of semantic domains, including colours, properties (old, small), group membership terms, and abstract concepts.

Many if not most instances of both patterns can be considered extravagant in the sense of Haspelmath (1999), although they arguably draw their extravagance from slightly different sources. While [the mother of all $\mathrm{X}]$ expresses a high degree of emotional involvement, [X BE the new $\mathrm{Y}]$ often involves creative and unconventional comparisons, some of which may be surprising because they are self-explanatory but not necessarily self-evident (sugar is the new nicotine), while others need an explicit elaboration of the tertium comparationis in order to be understood.

We have shown that the two patterns exhibit all three features that can be considered characteristic of snowclones: they can be traced back to a lexically fixed source constructions (or, in the case of [X BE the new Y], arguably to a family of source constructions, viz. fashion slogans of the type pink is the new black); they are used productively, but their productivity is constrained to certain domains; and they can be considered extravagant.

At the beginning of this paper, we have raised the question of whether the concept of snowclones is a useful one in the first place. We would argue that it is, as snowclones, despite sharing some of their features with other types of phrasal constructions, exhibit additional characteristics that set them apart from these patterns. For instance, we already discussed in Section 2 that snowclones bear some resemblance to phraseologisms but differ from the latter in virtue of their partial productivity and/or extravagant characteristics. Other examples of constructions that are partially similar to snowclones include items for which the German term 'Nervsprech' (roughly: 'annoying speech') has been proposed (Schulze 2013, Finkbeiner 2019). Examples include Tschüssikovsky (a blend of German tschüss 'bye' and Tchaikovsky), Schanke dön (instead of Danke schön 'Thank you'), or Der Apfel fällt nicht weit vom Pferd 'The apple doesn't fall far from the horse'. Like snowclones, 'Nervsprech' utterances are expressive and have a fairly clear lexically fixed source construction, but unlike snowclones, they are themselves relatively invariable.

While some phenomena are thus related but still clearly distinct from snowclones, the literature also contains examples of constructions that have been previously discussed on their own terms but which could be arguably re-classified as snowclones. Among these patterns are the 'expressive much' or 'sarcastic much' construction discussed by Gutzmann \& Henderson (2019) and Hilpert \& Bourgeois (2020), as in angry much?, and the 'expressive subordinate clause construction' discussed by Gutzmann \& Turgay (2019), as in German Der Moment wenn man checkt dass man verschlafen hat 'The moment you realise 
you've overslept'. Both of these patterns bear striking similarities to snowclones: they function as extravagant phrasal templates, and while they differ in the degree to which they do not seem to have a lexically fixed source construction from which they derive, they form a rather coherent group of constructions tied to specific discourse contexts (especially computer-mediated communication). As such, these constructions, but also snowclones, share important features with internet memes. Dancygier \& Vandelanotte (2017a) argue that internet memes draw their high degree of replicability from multiple factors, including constructional compositionality (i.e. the constructions keep their core meaning even when being adapted to very different contexts) and offering 'ways to profile emotional and experiential viewpoints on situations' (Dancygier \& Vandelanotte 2017a: 594).

Like internet memes, snowclones are highly malleable (or, in the words of Pullum's original definition quoted above, customisable) and can thus be adapted to a broad array of different situations. As with many other extravagant constructions (see e.g. Kempf \& Hartmann 2022), they allow language users to follow both the maxim of extravagance ('Talk in such a way that you are noticed') and the seemingly contradictory opposing maxim postulated by Haspelmath (1999: 1055) on the basis of Keller (1994), the maxim of conformity: 'talk like the others talk.' Some of the pop-cultural references on which snowclones are based may be opaque to many language users: for instance, while In space no one can hear you scream may still be relatively well-known, fewer people will be familiar with Breakin' 2: Electric Boogaloo. Snowclone users can thus identify as members of an in-group by showing that they are familiar with pop-cultural knowledge that belongs to the 'common ground' of the group, while at the same time being innovative and creative.

While we have focused on the definition as well as on semantic aspects of snowclones, there are still a number of open questions that should be addressed in future research. Firstly, it would be interesting to investigate the 'life course' of snowclones. In the case of internet memes, we often find short-lived trends (Bülow et al. 2018) - given the similarities pointed out above, the same could be expected for snowclones. Secondly, we have focused on snowclones in English, and for obvious reasons, it would be worthwhile to take other languages into account as well. The influence of language contact could also be considered in this context. In German, for example, we find equivalents of the two snowclones discussed in the present paper. This raises the question to what extent the inventory of snowclones in a given language is adapted from English, which can probably be considered the lingua franca of contemporary pop culture. Thirdly, it is an open question to what extent snowclones are tied to (certain text types of) written or spoken language, and there is no reason not to assume that snowclones can also be found in signed languages. Last but not least, it seems worthwhile to study the relationship between snowclones and other extravagant and/or expressive constructions more systematically. We have already pointed to some commonalities between snowclones and several constructions or construction families that have been discussed as extravagant, but we expect 
that other similarities, for example with regard to usage contexts and text type distributions, could also play a relevant role.

In this paper, we have illustrated that speakers' use of snowclones and other extravagant formulaic expressions can be investigated with the help of systematic criteria and quantitative corpus-based tools. We hope that our approach and methods will motivate other studies of how speakers' creativity and desire to be noticed shape current linguistic practices.

\section{DATA AVAILABILITY STATEMENT}

The datasets and scripts used for the analysis reported on in the present paper can be found here: https://osf.io/97t54/?view_only=63aa9f82be79412a895d4749a77 $5 \mathrm{~b} 758$ (anonymised for review).

\section{THE MOTHER OF ALL ACKNOWLEDGMENTS}

(to be added after review)

\section{COMPETING INTERESTS DECLARATION}

The authors declare none.

\section{REFERENCES}

Bergs, Alexander. 2018. Learn the Rules like a Pro, so you can Break them like an Artist (Picasso): Linguistic Aberrancy from a Constructional Perspective. Zeitschrift für Anglistik und Amerikanistik 66(3). 277-293. doi:10.1515/zaa-2018-0025. https://www.degruyter.com/document/doi/10.1515/ zaa-2018-0025/html. Publisher: De Gruyter.

Bergs, Alexander. 2019. What, if anything, is linguistic creativity? Gestalt Theory 41(2). 173-183. doi:10.2478/gth-2019-0017. https://content.sciendo.com/view/journals/gth/41/2/article-p173.xml.

Booij, Geert. 2002. Constructional Idioms, Morphology, and the Dutch Lexicon. Journal of Germanic Linguistics 14(4). 301-327.

Bülow, Lars, Marie-Luis Merten \& Michael Johann. 2018. Internet-Memes als Zugang zu multimodalen Konstruktionen. Zeitschrift für Angewandte Linguistik 2018(69). 1-32. doi: 10.1515/zfal-2018-0015. http://www.degruyter.com/view/j/zfal.2018.69.issue-1/zfal-2018-0015/ zfal-2018-0015.xml.

Culicover, Peter W. \& Ray Jackendoff. 1999. The View from the Periphery: The English Comparative Correlative. Linguistic Inquiry 30(4). 543-571. doi:10.1162/002438999554200. https://doi.org/10. 1162/002438999554200.

Cummins, Chris. 2019. Pragmatics. Edinburgh: Edinburgh University Press.

Dammerer, Emmanuel Maria. 2007. Buch von der deutschen Snowclonerey. http://emmanuel. dammerer.at/snowclonerey.

Dancygier, Barbara \& Lieven Vandelanotte. 2017a. Internet memes as multimodal constructions. Cognitive Linguistics 28(3). 565-598. doi:10.1515/cog-2017-0074. http://www.degruyter.com/ view/j/cogl.2017.28.issue-3/cog-2017-0074/cog-2017-0074.xml.

Dancygier, Barbara \& Lieven Vandelanotte. 2017b. Reappraising 'snowclones': Replicability and Construction Grammar, Tartu, Estonia.

Davies, Mark. 2008. The Corpus of Contemporary American English (COCA). https://www. english-corpora.org/coca/.

Davies, Mark. 2010. The Corpus of Historical American English (COHA). https://www. english-corpora.org/coha/. 
De Wit, Astrid, Peter Petré \& Frank Brisard. 2020. Standing out with the progressive. Journal of Linguistics 1-36. doi:10.1017/S0022226719000501. https://www.cambridge.org/core/product/ identifier/S0022226719000501/type/journal_article.

Dobrovol'skij, Dimitrij. 2013. Phraseologie und Konstruktionsgrammatik. In Alexander Ziem \& Alexander Lasch (eds.), Konstruktionsgrammatik: Konzepte und Grundlagen gebrauchsbasierter Ansätze, 111-130. Berlin, New York: De Gruyter.

Eitelmann, Matthias \& Dagmar Haumann (eds.). 2022. Extravagant morphology. Amsterdam, Philadelphia: John Benjamins.

Fauconnier, Gilles \& Mark Turner. 2002. The Way We Think: Conceptual Blending and the Mind's Hidden Complexities. New York: Basic Books.

Ferguson, Kevin L. 2019. Pop goes the decade. The nineties. Santa Barbara, California: Greenwood first edition edn.

Fillmore, Charles J., Paul Kay \& Mary Catherine O’Connor. 1988. Regularity and Idiomaticity in Grammatical Constructions: The Case of Let Alone. Language 64(3). 501-538. doi:10.2307/ 414531. https://www.jstor.org/stable/414531. Publisher: Linguistic Society of America.

Finkbeiner, Rita. 2019. Tschüssikowski und Bis später, Attentäter. Zur Bedeutung von expressiven Verabschiedungen. In Franz-Josef d'Avis \& Rita Finkbeiner (eds.), Expressivität im Deutschen (Reihe Germanistische Linguistik 318), 341-369. Berlin, Boston: De Gruyter.

Flach, Susanne. 2017. collostructions: An R Implementation for the Family of Collostructional Methods. www.bit.ly/sflach.

Glucksberg, Sam \& Boaz Keysar. 1993. How metaphors work. In Andrew Ortony (ed.), Metaphor and Thought, 401-424. Cambridge: Cambridge University Press 2nd edn.

Grady, Joseph E. 1999. A typology of motivation for conceptual metaphor: Correlation vs. resemblance. In Raymond W. Gibbs \& Gerard J. Steen (eds.), Metaphor in cognitive linguistics: selected papers from the Fifth International Cognitive Linguistics Conference, Amsterdam, July 1997, 79-100. Amsterdam: Benjamins. OCLC: 248784237.

Gries, Stefan Th. 2019. 15 years of collostructions: Some long overdue additions/corrections (to/of actually all sorts of corpus-linguistics measures). International Journal of Corpus Linguistics 24(3). 385-412. doi:10.1075/ijcl.00011.gri. http://www.jbe-platform.com/content/journals/10.1075/ijcl. 00011.gri.

Gutzmann, Daniel \& Robert Henderson. 2019. Expressive updates, much? Language 95(1). 107-135. doi:10.1353/lan.2019.0014. https://muse.jhu.edu/article/719236.

Gutzmann, Daniel \& Katharina Turgay. 2019. Der Moment, wenn dir klar wird, dass es expressive Nebensätze gibt. Zur Syntax und Semantik der expressiven Nebensatzkonstruktion. In Franz-Josef d'Avis \& Rita Finkbeiner (eds.), Expressivität im Deutschen (Reihe Germanistische Linguistik 318), 261-291. Berlin, Boston: De Gruyter.

Hartmann, Stefan. 2014. Constructing a Schema: Word-Class Changing Morphology in a UsageBased Perspective. In Martin Hilpert \& Susanne Flach (eds.), Yearbook of the German Cognitive Linguistics Association, Vol. 2, 235-252. Berlin, New York: De Gruyter.

Haspelmath, Martin. 1999. Why is grammaticalization irreversible? Linguistics 37(6). 1043-1068. doi:10.1515/ling.37.6.1043. http://www.degruyter.com/view/j/ling.1999.37.issue-6/ling.37.6.1043/ ling.37.6.1043.xml.

Hill, Ian E. J. 2018. Memes, munitions, and collective copia: The durability of the perpetual peace weapons snowclone. Quarterly Journal of Speech 104(4). 422-443. doi:10.1080/00335630.2018. 1519255. https://www.tandfonline.com/doi/full/10.1080/00335630.2018.1519255.

Hilpert, Martin \& Samuel Bourgeois. 2020. Intersubjectification in constructional change: From confrontation to solidarity in the sarcastic much? construction. Constructions and Frames 12(1). 96-120. doi:10.1075/cf.00036.hil. http://www.jbe-platform.com/content/journals/10.1075/ cf.00036.hil.

Hilpert, Martin \& Florent Perek. 2015. Meaning change in a petri dish: constructions, semantic vector spaces, and motion charts. Linguistics Vanguard 1(1). 339-350.

Howarth, Peter Andrew. 1996. Phraseology in English Academic Writing: Some implications for language learning and dictionary making. Tübingen: Niemeyer. OCLC: 1154240198.

Kay, Paul \& Charles J. Fillmore. 1999. Grammatical constructions and linguistic generalizations: The What's X doing Y? construction. Language 75(1). 1-33. doi:10.1353/lan.1999.0033.

Keller, Rudi. 1994. Sprachwandel: Von der unsichtbaren Hand in der Sprache. Tübingen, Basel: Francke.

Kempf, Luise \& Stefan Hartmann. 2022. What's extravagant about be-sandal-ed feet? Extravagance and productivity of German pseudo-participles. In Matthias Eitelmann \& Dagmar Haumann (eds.), Extravagant morphology, Amsterdam, Philadelphia: John Benjamins. 
Lass, Roger. 2015. Lineage and the constructive imagination. The birth of historical linguistics. In Claire Bowern \& Bethwyn Evans (eds.), The Routledge handbook of historical linguistics, 45-63. New York: Routledge.

Levshina, Natalia. 2015. How to do linguistics with R. Data exploration and statistical analysis. Amsterdam, Philadelphia: John Benjamins.

Levshina, Natalia \& Kris Heylen. 2014. A Radically Data-driven Construction Grammar: Experiments with Dutch causative constructions. In Ronny Boogaart, Timothy Colleman \& Gijsbert Rutten (eds.), Extending the Scope of Construction Grammar, Berlin, New York: De Gruyter.

O'Connor, Erin. 2007. The snowclones database. http://itre.cis.upenn.edu/ myl/languagelog/archives/ 002806.html.

Perek, Florent. 2016. Using distributional semantics to study syntactic productivity in diachrony. A case study. Linguistics 54(1). 149-188.

Perek, Florent \& Martin Hilpert. 2017. A distributional semantic approach to the periodization of change in the productivity of constructions. International Journal of Corpus Linguistics 22(4). 490-520. doi:10.1075/ijcl.16128.per. http://www.jbe-platform.com/content/journals/10.1075/ijcl. 16128.per.

Petré, Peter. 2017. The extravagant progressive: an experimental corpus study on the history of emphatic be V-ing. English Language and Linguistics 21(2). 227-250. doi:10.1017/ S1360674317000107.

Pullum, Geoffrey K. 2003a. Bleached conditionals. http://itre.cis.upenn.edu/ myl/languagelog/ archives/000049.html.

Pullum, Geoffrey K. 2003b. Phrases for lazy writers in kit form. http://itre.cis.upenn.edu/ myl/ languagelog/archives/000061.html.

Pullum, Geoffrey K. 2004. Snowclones: Lexicographical dating to the second. http://itre.cis.upenn. edu/ myl/languagelog/archives/000350.html.

R Core Team. 2020. R: A language and environment for statistical computing. manual Vienna, Austria. https://www.R-project.org/. Tex.organization: R Foundation for Statistical Computing.

Sailer, Manfred. 2013. Idiom and Phraseology. Tech. rep. Oxford University Press. doi:10.1093/obo/ 9780199772810-0137. https://oxfordbibliographies.com/view/document/obo-9780199772810/ obo-9780199772810-0137.xml. Type: dataset.

Schmid, Hans-Jörg \& Helmut Küchenhoff. 2013. Collostructional Analysis and other Ways of Measuring Lexicogrammatical Attraction: Theoretical Premises, Practical Problems and Cognitive Underpinnings. Cognitive Linguistics 24(3). 531-577.

Schulze, Florian. 2013. Noch so 'ne Phrase, Faust auf die Nase! Eine phraseologische Untersuchung des Nerv-Sprech. Linguistik Online 60(3).

Schäfer, Roland. 2015. Processing and querying large web corpora with the COW14 architecture. In Piotr Baski, Hanno Biber, Evelyn Breiteneder, Marc Kupietz, Harald Lüngen \& Andreas Witt (eds.), Proceedings of challenges in the management of large corpora 3 (CMLC-3), Lancaster: IDS. http://rolandschaefer.net/?p=749. Tex.organization: UCREL.

Schäfer, Roland \& Felix Bildhauer. 2012. Building Large Corpora from the Web Using a New Efficient Tool Chain. In Nicoletta Calzolari, Khalid Choukri, Terry Declerck, Mehmet Uur Doan, Bente Maegaard, Joseph Mariani, Asuncion Moreno, Jan Odijk \& Stelios Piperidis (eds.), Proceedings of LREC 2012, 486-493.

Smirnova, Elena. 2021. Deutsche Partizipialkomposita aus diachroner Perspektive. In Christine Ganslmayer \& Christian Schwarz (eds.), Historische Wortbildung: Theorie - Methode Perspektiven, 383-415. Hildesheim: Olms.

Stefanowitsch, Anatol. 2013. Collostructional Analysis. In Thomas Hoffmann \& Graeme Trousdale (eds.), The Oxford Handbook of Construction Grammar, 290-306. Oxford: Oxford University Press.

Stefanowitsch, Anatol \& Stefan Th. Gries. 2003. Collostructions: Investigating the Interaction of Words and Constructions. International Journal of Corpus Linguistics 8(2). 209-243.

Stefanowitsch, Anatol \& Stefan Th Gries. 2005. Covarying Collexemes. Corpus Linguistics and Linguistic Theory 1(1). 1-43.

Tizón Couto, David. 2021. The rise of COVID snowclones the mother of all linguistic phrases. https:// theconversation.com/the-rise-of-covid-snowclones-the-mother-of-all-linguistic-phrases-167580.

Traugott, Elizabeth Closs. 2014. Towards a Constructional Framework for Studying Language Change. Cognitive Linguistic Studies 1(1). 3-21.

Traugott, Elizabeth Closs \& Graeme Trousdale. 2013. Constructionalization and Constructional Changes (Oxford Studies in Diachronic and Historical Linguistics 6). Oxford: Oxford University Press. 
Traugott, Elizabeth Closs \& Graeme Trousdale. 2014. Contentful constructionalization. Journal of Historical Linguistics 4(2). 256-283. doi:10.1075/jhl.4.2.04tra. http://www.jbe-platform.com/ content/journals/10.1075/jhl.4.2.04tra.

Ungerer, Tobias \& Stefan Hartmann. 2020. Delineating extravagance: Assessing speakers' perceptions of imaginative constructional patterns. Belgian Journal of Linguistics 34. 345-356. doi:10.1075/ bj1.00058.ung. https://benjamins.com/catalog/bj1.00058.ung.

Wheeler, Eric S. 2005. Multidimensional Scaling for Linguists. In Reinhard Köhler, Gabriel Altmann \& Rajmund G. Piotrowski (eds.), Quantitative Linguistics (HSK 27), 548-553. Berlin, New York: De Gruyter.

Zimmer, Ben. 2007. Phrasal patterns 2: Electric boogaloo. https://blog.oup.com/2007/08/patterns/.

Zimmer, Benjamin. 2006. On the trail of the new black (and the navy blue). http://itre.cis.upenn.edu/ $\sim$ myl/languagelog/archives/003981.html.

Zwicky, Arnold. 2006. Fully awesome! http://itre.cis.upenn.edu/\%7emyl/languagelog/archives/ 003764.html.

Zwicky, Arnold. 2007. Snowclone mountain? http://itre.cis.upenn.edu/ myl/languagelog/archives/ 002924.html.

The snowclones will return in 'Attack of the snowclones 2: Electric boogaloo'.

Authors' addresses: (Hartmann)

University of Düsseldorf, Universitätsstraße 1, 40225 Düsseldorf,

Germany

hartmast@hhu.de

(Ungerer)

University of Edinburgh, Dugald Stewart Building, 3 Charles

Street, Edinburgh, EH8 9AD, UK

t.ungerer@ed.ac.uk 